\title{
Introduction to Focus Issue: Dynamics in Systems Biology
}

\author{
Chris A. Brackley, ${ }^{1, a)}$ Oliver Ebenhöh, ${ }^{2}$ Celso Grebogi, ${ }^{2}$ Jürgen Kurths, ${ }^{2}$ \\ Alessandro de Moura, ${ }^{2}$ M. Carmen Romano, ${ }^{2}$ and Marco Thiel ${ }^{2}$ \\ ${ }^{1}$ Institute for Complex Systems and Mathematical Biology, SUPA King's College, University of Aberdeen, \\ Aberdeen AB24 3UE, United Kingdom \\ ${ }^{2}$ Institute for Complex Systems and Mathematical Biology, SUPA, University of Aberdeen, \\ Aberdeen AB24 3UE, United Kingdom
}

(Received 1 December 2010; published online 30 December 2010)

\begin{abstract}
The methods of nonlinear systems form an extensive toolbox for the study of biology, and systems biology provides a rich source of motivation for the development of new mathematical techniques and the furthering of understanding of dynamical systems. This Focus Issue collects together a large variety of work which highlights the complementary nature of these two fields, showing what each has to offer the other. While a wide range of subjects is covered, the papers often have common themes such as "rhythms and oscillations," "networks and graph theory," and "switches and decision making." There is a particular emphasis on the links between experimental data and modeling and mathematical analysis. (C) 2010 American Institute of Physics. [doi:10.1063/1.3530126]
\end{abstract}

\begin{abstract}
Nonlinear dynamical systems can be found throughout biology. This Focus Issue showcases examples of work where mathematical approaches have successfully been applied to a myriad of biological problems. From the processing and understanding of the vast amounts of experimental data which are the fruits of recent advances in measurement techniques, to modeling exploits which motivate developments in mathematics, presented here are some highly interesting contributions to this blossoming field of systems biology.
\end{abstract}

The biological sciences have experienced a dramatic change over the past decades. With the rapid advance of high-throughput technologies, it has now become possible to simultaneously monitor many molecular components of a cell with an unprecedented temporal and spatial resolutionthus rendering molecular biology a truly quantitative science. Despite this technological advance, the interpretation and condensation of the immense amount of available data still pose major challenges, and overarching theories of general validity are still sparse.

The necessity to develop new theories and to describe biological systems with mathematical methods is reflected in the rapidly growing scientific field of systems biology. Many different approaches are being developed and the diversity of investigated systems mirrors the versatility of the life sciences. From a physicist's point of view, living organisms can be understood as highly complex dynamic systems with various levels of organization, all of which influence each other. However, to view life as a special instance of physics does not fully account for the true complexity of biology. Most importantly, the biological systems that we observe today have been shaped (and are still being shaped) by billions of years of evolutionary pressure. The mere fact that these systems exist today reflects their success in the everlasting

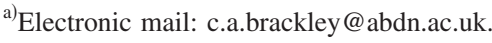

struggle for survival. Indeed, survival in this constantly changing, harsh, and competitive environment requires special features which one would not expect to have appeared purely by chance.

Despite the advances in biological measurement technologies, the available data are by far less complete than for physical systems, such as astronomical bodies or electronic devices. This necessitates a completely different approach to data analysis. To unravel the mysteries of biology and give our myriad of observations a unifying theoretical superstructure, it is clearly required that life is investigated on all spatial, temporal, and organizational levels and that common patterns, or evolutionary design principles, are identified. Considering our limited knowledge, it is evident that we are presently still at the very beginning of a new and fascinating era of theoretical biological research with unpredictable outcome.

An important contribution to the development of general biological theories is certainly the study of the dynamics of biological systems, on scales ranging from short-term adaptive processes which occur in seconds or faster to evolutionary dynamics which extend over billions of years. The purpose of this Focus Issue is to bring together work from the many diverse areas where the techniques of dynamical system research are applied to biology. Nonlinear dynamics offers the possibility to structure the data and to make sense of the rich experimental results. The complexity of biological organisms and the intricate interactions between different subsystems make it necessary to use cutting-edge mathematics to unravel the mechanisms that lie at the heart of life.

Different areas of dynamical systems theory, such as synchronization and network theory, offer suitable tools for the analysis and description of biological systems and cast new light on experimental data and their interpretation. This systematic, quantitative, and predictive approach characterizes the increasingly important interplay between the biological and mathematical sciences. Crossing the border between these formerly separated branches, systems biology fosters a cross-fertilization of ideas and stimulates new thinking, 
opening a treasure trove of novel problems which have been unnoticed until recently.

It is becoming more and more obvious that the theory of dynamical systems and chaos is key for the growth of biological knowledge in the coming years. This Focus Issue shows some examples of a successful application of various mathematical concepts to diverse biological systems. It suggests how the expected revolution in our understanding of biological systems might, like other scientific developments before, be triggered by new mathematics and the interaction of scientists from different disciplines.

Although the range of subjects is deliberately wide, there are several core themes which are repeatedly addressed from different angles.

One vital facet of all biological systems is the ability to generate rhythmicity and oscillations: from the smallest single celled organism to the complexity of our own bodies, life relies on cycles and timing. The beating of the human heart is analyzed by Thul and Coombes, ${ }^{1}$ who studied a model of intercellular calcium dynamics in order to understand cardiac alternans-a precursor to fibrillation. Also concerned with fibrillation, Petrov et $a l^{2}{ }^{2}$ examined spiral wave stability and the influence of fibroblasts. Harvey et $a .^{3}$ considered calcium dynamics on an intracellular length scale. They use recently developed canard theory for systems with three or more slow variables to study models of calcium dynamics. Raue et al. ${ }^{4}$ studied identifiability and observability analysis of experimental data, considering how such techniques can inform experimental and model design.

Signaling and oscillations are also at the heart of neural systems; Chandrasekar et al. ${ }^{5}$ represented neuronal populations as populations of coupled oscillators in order to understand the mechanisms of synchronization - an often pathological phenomena associated with seizures. Finke ${ }^{6}$ revisited the classic Hodgekin-Huxley model of a neuron to study the mechanism of cold reception in mammals. On smaller length scale oscillators can be used as cellular clocks: Morant et al. ${ }^{7}$ examined the circadian clock in green algae, analyzing time series data which shows remarkable agreement with models. Gérard et $_{\mathrm{al}}{ }^{8}$ studied a network of kinases which control the dynamics of the mammalian cell cycle. They showed that as a result of multiple oscillatory network circuits, there exists rich dynamics such as complex periodic orbits, quasiperiodic oscillations, and chaos.

Networks and graph theory are themselves important tools in systems biology. In particular, protein interaction networks can be used to understand the many complex interactions between different proteins in a cell, for example, in how a cell responds to extracellular signals. Rue et al. ${ }^{9}$ studied the signal transduction network found in human fibroblasts; they use a simple Boolean representation of the network with experimentally motivated parameters to show that the relaxation to an attractor is robust to noise. Koseska and Kurths ${ }^{10}$ considered network subunits (such as switches and oscillators) in synthetic biological systems. They studied how the addition of such circuits in a network can enhance the presence of different dynamical regimes. Interesting network structures arise in many biological applications: Zhandov ${ }^{11}$ considered a layered network model of mRNAprotein interactions, including noncoding RNAs. Wan et al. ${ }^{12}$ studied a network growth model, looking at how a network evolves based on simple rules, with the aim of producing a structure which reflects the topology of a real protein network. Wang et al. ${ }^{13}$ examined the evolution of functional subnetworks. Network theory not only allows the study of the evolution of networks themselves but can also be used to further the understanding of evolution in biology. Schütte et $a l .{ }^{14}$ studied evolutionary dynamics of metabolic pathways; they show, by considering evolutionary walks on the metabolic network, that new enzymes appear in clusters rather than gradually.

Evolution is another area where mathematical methods are crucial to furthering our understanding. Ni et al. ${ }^{15} \mathrm{de}-$ scribed how the traditional "rock-paper-scissors" game can be used to understand the dynamics of species coexistence on evolutionary time scales. They showed that from simple competition rules, different basins of attraction (e.g., coexistence or extinction) can emerge. Slipantschuk et al. ${ }^{16}$ showed that not only can dynamical systems teach us about biology but also the inverse is true: they gain understanding of chaotic systems from a study of a grouse population dynamics model with delay differential equations. They find that the formation of shrimp shaped periodic regimes in parameter space is triggered by a homoclinic bifurcation.

Evolutionary decisions are not the only decisions that biology has to make. On shorter length and time scales, cells and organisms have many choices to make based on sensory input and previous experience. Neri ${ }^{17}$ mathematically treated human sensory processing as a mapping between a stimulus vector and a decision variable. Learning and adaption are examined by Komarov et al. ${ }^{18}$ who studied a model consisting of a network of nonlinear dynamical elements which produce sequences of goal directed actions.

Another feature of some dynamical systems which leads to decision-making-like functionality is bistability; bistable switches are very prevalent in biology. Cellular decision making is studied by Domingo-Sananes and Novak, ${ }^{19}$ who focused on a biochemical regulatory network which controls a transition in the cell cycle. They highlighted the difference between positive and double negative feedbacks and how differences in architecture can change the position of the saddle node bifurcation which determines where the system switches. Schittler et al. ${ }^{20}$ considered a model of a two switch regulatory genetic network applied to differentiation of stem cells. They are able to reproduce three experimentally observed equilibrium states.

Dynamical systems also play a role in understanding mechanical processes in biology. Günther and Kruse ${ }^{21}$ studied force generation in muscles, focusing on the dynamics of sarcomeres - the basic force generating subunits. They analyzed Hopf bifurcations, canard explosions, and gluing bifurcations and considered the implications for experiments. Blood flow is studied by Geddes et al., ${ }^{22}$ who examined the topology of microvascular networks. They found multiple steady states due to the nonlinear dependence of viscosity on blood cell concentration and presented evidence of how the predicted phenomena can be observed experimentally. Suhrbier et al. $^{23}$ examined the role of the cardiovascular system in sleep. They present a method for the detection of timedelayed coupling in time series, applied to heat rate and blood pressure data during different stages of sleep. 
Dynamical systems also have a role to play in medical applications. Hirata et al. ${ }^{24}$ presented a mathematical model for the treatment of cancer. The method aims to find an optimal protocol for intermittent drug delivery, using mathematics to inform physicians on how best to treat individual patients.

The guest editors would like to thank all of the authors who contributed to this Focus Issue. Also, we are grateful to Janis Bennett (Assistant Editor, Chaos) for much help in the logistics of putting together this issue.

${ }^{1} \mathrm{R}$. Thul and S. Coombes, "Understanding cardiac alternans: A piecewise linear modelling framework," Chaos 20, 045102 (2010).

${ }^{2}$ V. S. Petrov, G. V. Osipov, and J. Kurths, "Fibroblasts alter spiral wave stability," Chaos 20, 045103 (2010).

${ }^{3}$ E. Harvey, V. Kirk, H. M. Osinga, J. Sneyd, and M. Wechselberger, "Understanding anomalous delays in a model of intracellular calcium dynamics," Chaos 20, 045104 (2010).

${ }^{4}$ A. Raue, V. Becker, U. Klingmüller, and J. Timmer, "Identifiability and observability analysis for experimental design in nonlinear dynamical models," Chaos 20, 045105 (2010).

${ }^{5}$ V. K. Chandrasekar, J. H. Sheeba, and M. Lakshmanan, "Pathological mass synchronization in the brain: Occurrence and its control," Chaos 20 , 045106 (2010).

${ }^{6}$ C. Finke, J. A. Freund, E. Rosa, H. Braun, and U. Feudel, "On the role of subthreshold currents in the Huber-Braun cold receptor model," Chaos 20 , 045107 (2010).

${ }^{7}$ P. Morant, Q. Thommen, B. Pfeuty, C. Vandermoere, F. Corellou, F-Y Bouget, and M. Lefranc, "A robust two-gene oscillator at the core of Ostreococcus tauri circadian clock," Chaos 20, 045108 (2010).

${ }^{8} \mathrm{C}$. Gérard and A. Goldbeter, "From simple to complex patterns of oscillatory behavior in a model for the mammalian cell cycle containing multiple oscillatory circuits," Chaos 20, 045109 (2010).

${ }^{9}$ P. Rué, T. Pons, N. Domedel-Puig, and J. Garcia-Ojalvo, "Relaxation dynamics and frequency response of a noisy cell signaling network," Chaos 20, 045110 (2010).
${ }^{10}$ A. Koseska and J. Kurths, "Topological structures enhance the presence of dynamical regimes in synthetic networks," Chaos 20, 045111 (2010).

${ }^{11}$ V. P. Zhdanov, "Hierarchical genetic networks and non-coding RNAs," Chaos 20, 045112 (2010)

${ }^{12}$ X. Wan, S. M. Cai, J. Zhou, and Z. Liu, "Emergence of modularity and disassortativity in protein-protein interaction networks," Chaos $\mathbf{2 0}$, 045113 (2010).

${ }^{13} \mathrm{M}$. Li, X. Wang, and C. Lai, "Evolution of functional subnetworks in complex systems," Chaos 20, 045114 (2010).

${ }^{14}$ M. Schütte, A. Skupin, D. Segre, and O. Ebenhöh, "Modeling the complex dynamics of enzyme-pathway coevolution," Chaos 20, 045115 (2010).

${ }^{15} \mathrm{X}$. Ni, W. Wang, R. Yang, Y.-C. Lai, and C. Grebogi, "Basins of coexistence and extinction in spatially extended ecosystems of cyclically competing species," Chaos 20, 045116 (2010).

${ }^{16}$ J. Slipantschuk and E. Ullner, M. da silva Baptista, M. Zeineddine, and M. Thiel, "Abundance of stable periodic behavior in a Red Grouse population model with delay: A consequence of homoclinicity," Chaos 20, 045117 (2010).

${ }^{17} \mathrm{P}$. Neri, "Stochastic characterization of small-scale algorithms for human sensory processing," Chaos 20, 045118 (2010).

${ }^{18}$ M. Komarov, G. V. Osipov, and M. S. Burtsev, "Adaptive functional systems: Learning with chaos," Chaos 20, 045119 (2010).

${ }^{19}$ M. R. Domingo-Sananes and B. Novak, "Different effects of redundant feedback loops on a bistable switch," Chaos 20, 045120 (2010).

${ }^{20}$ D. Schittler and J. Hasenauer, F. Allg Ãwer, and S. Waldherr, "Cell differentiation modeled via a coupled two-switch regulatory network," Chaos 20, 045121 (2010).

${ }^{21}$ S. Günther and K. Kruse, "Spontaneous sarcomere dynamics," Chaos 20 , 045122 (2010).

${ }^{22}$ J. Geddes, R. Carr, F. Wu, Y. Lao, and M. Maher, "Blood flow in microvascular networks: A study in nonlinear biology," Chaos 20, 045123 (2010).

${ }^{23}$ A. Suhrbier, M. Riedl, H. Malberg, T. Penzel, G. Bretthauer, J. Kurths, and N. Wessel, "Cardiovascular regulation during sleep quantified by symbolic coupling traces," Chaos 20, 045124 (2010).

${ }^{24}$ Y. Hirata, M. Di Bernardo, N. Bruchovsky, and K. Aihara, "Hybrid optimal scheduling for intermittent androgen suppression of prostate cancer," Chaos 20, 045125 (2010). 